Ocean Sci., 6, 431-439, 2010

www.ocean-sci.net/6/431/2010/

(C) Author(s) 2010. This work is distributed under

the Creative Commons Attribution 3.0 License.

\title{
First images and orientation of fine structure from a 3-D seismic oceanography data set
}

\author{
T. M. Blacic and W. S. Holbrook \\ University of Wyoming, Geology and Geophysics Department, 1000 E. University Ave., Laramie, WY 82071, USA
}

Received: 11 September 2009 - Published in Ocean Sci. Discuss.: 20 October 2009

Revised: 11 February 2010 - Accepted: 29 March 2010 - Published: 20 April 2010

\begin{abstract}
We present 3-D images of ocean fine structure from a unique industry-collected 3-D multichannel seismic dataset from the Gulf of Mexico that includes expendable bathythermograph casts for both swaths. 2-D processing reveals strong laterally continuous reflections throughout the upper $\sim 800 \mathrm{~m}$ as well as a few weaker but still distinct reflections as deep as $\sim 1100 \mathrm{~m}$. We interpret the reflections to be caused by reversible fine structure from internal wave strains. Two bright reflections are traced across the $225-\mathrm{m}-$ wide swath to produce reflection surface images that illustrate the 3-D nature of ocean fine structure. We show that the orientation of linear features in a reflection can be obtained by calculating the orientations of contours of reflection relief, or more robustly, by fitting a sinusoidal surface to the reflection. Preliminary 3-D processing further illustrates the potential of 3-D seismic data in interpreting images of oceanic features such as internal wave strains. This work demonstrates the viability of imaging oceanic fine structure in 3-D and shows that, beyond simply providing a way visualize oceanic fine structure, quantitative information such as the spatial orientation of features like fronts and solitons can be obtained from 3-D seismic images. We expect complete, optimized 3-D processing to improve both the signal to noise ratio and spatial resolution of our images resulting in increased options for analysis and interpretation.
\end{abstract}

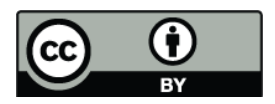

Correspondence to: T. M. Blacic (tblacic@uwyo.edu)

\section{Introduction}

Ocean mixing processes are fundamentally 3-D in nature and vary in time on a wide range of scales. Features that affect thermohaline fine structure, such as internal waves, tidal beams, solitons, eddies, fronts, warm core rings, and turbulent patches, are expected to vary in both space and time. To study these kinds of features, oceanographers typically use a combination of surface measurements (e.g., satellite sea surface elevation and surface temperature; Egbert et al., 1994; Ikeda and Emery, 1984), vertical profiles (by expendable instruments, non-expendable lowered instruments, or moored instrument arrays; e.g., Cooper et al., 1990; Rudnick et al., 2003), and towed instruments that can take measurements either along one horizontal line or in a "tow-yo" sawtooth pattern (e.g., Rudnick and Ferrari, 1999; Klymak and Moum, 2007). These methods can capture large-scale patterns over a wide area or fine-scale patterns at a discrete location or depth. High frequency ( $100 \mathrm{kHz}$ to $1 \mathrm{MHz}$ ) sonar methods can provide 2-D images of backscattering in the upper ocean, which are interpreted to result from zooplankton, suspended sediment or sound-speed microstructure, and are used to map internal waves at shallow depths (e.g., Wiebe et al., 1997; Farmer and Armi, 1999). This array of measurement techniques leaves a large portion of the ocean (i.e., depths below a few hundred meters) under-sampled both laterally and vertically and opens the door to new ways of mapping oceanic fine structure.

Recently, a new method for studying the ocean has emerged called seismic oceanography. In this method, scientists process conventional low frequency $(10$ to $100 \mathrm{~Hz})$ multichannel seismic (MCS) data to focus on imaging reflections in the water column instead of below the sea floor

Published by Copernicus Publications on behalf of the European Geosciences Union. 
(Holbrook et al., 2003). The reflections in the water column have been shown by Nandi et al. (2004) to primarily arise from water temperature fluctuations as small as $0.03^{\circ} \mathrm{C}$. Although the images obtained can be intrinsically revealing of ocean structure, concurrent hydrographic measures (temperature/salinity profiles) are needed to ground truth the temperature variations highlighted by the reflections. Thus far, only 2-D seismic profiles have been processed for seismic oceanography (e.g., Holbrook et al., 2003; Tsuji et al., 2005; Nakamura et al., 2006; Biescas et al., 2008), although it is now standard in the oil industry to collect 3-D seismic data using ships that tow up to seventeen parallel hydrophone streamers. Here we present the first 3-D images of ocean fine structure obtained from a data set collected by a large oil company that includes concurrent expendable bathythermograph (XBT) profiles. This industry data set may be the first of its kind with coincident seismic and water temperature measurements and represents an important step towards increasing cooperation and data sharing with the oil industry.

\section{Methods}

The data set consists of two 8-cable, 2-source 3-D swaths in the Gulf of Mexico off the coast of Texas that overlap in space and are separated in time by approximately two days. Swath S168, $20.3 \mathrm{~km}$ in length, was collected on 2 February 2006, and swath $S 170,19.0 \mathrm{~km}$ in length, was collected on 4 February 2006. Both were collected from NW to SE (Fig. 1). The seismic ship towed an array of 8 streamers spaced $60 \mathrm{~m}$ apart, each $4.65 \mathrm{~km}$ in length. Channel spacing along the streamers was $12.5 \mathrm{~m}$; source spacing was $25 \mathrm{~m}$ for both sources with a flip-flop pattern $(50 \mathrm{~m}$ when a single source is considered). This geometry results in common midpoint traces (CMPs) spaced every $6.25 \mathrm{~m}$, representing the horizontal sample spacing of the resulting image in the along-swath direction. Across-swath sample spacing is determined by the source and receiver configuration. When each source is considered separately the sampled swath is $225 \mathrm{~m}$ wide, with an across-swath sample spacing of $15 \mathrm{~m}$.

Eight expendable bathythermographs (XBTs) were deployed for each swath (Fig. 1). Casts were made from a second ship following the tail buoys at the end of the hydrophone streamers towed by the seismic vessel. The XBTs were launched to coincide with certain shot points in the seismic data in order to achieve a uniform spacing. Temperature profiles for the XBTs coinciding with swath S170 are shown in Fig. 2.

For a first look at our 3-D data set we focus on swath S170, processing each of the eight cables as individual 2D lines for each of the two sources, thus yielding 16 parallel seismic sections (images). To mitigate the effect of streamer feathering we only used the near 32 traces for each shot-cable pair. This results in a stacking fold of 4 and images that are noisier compared to full-fold images. However,
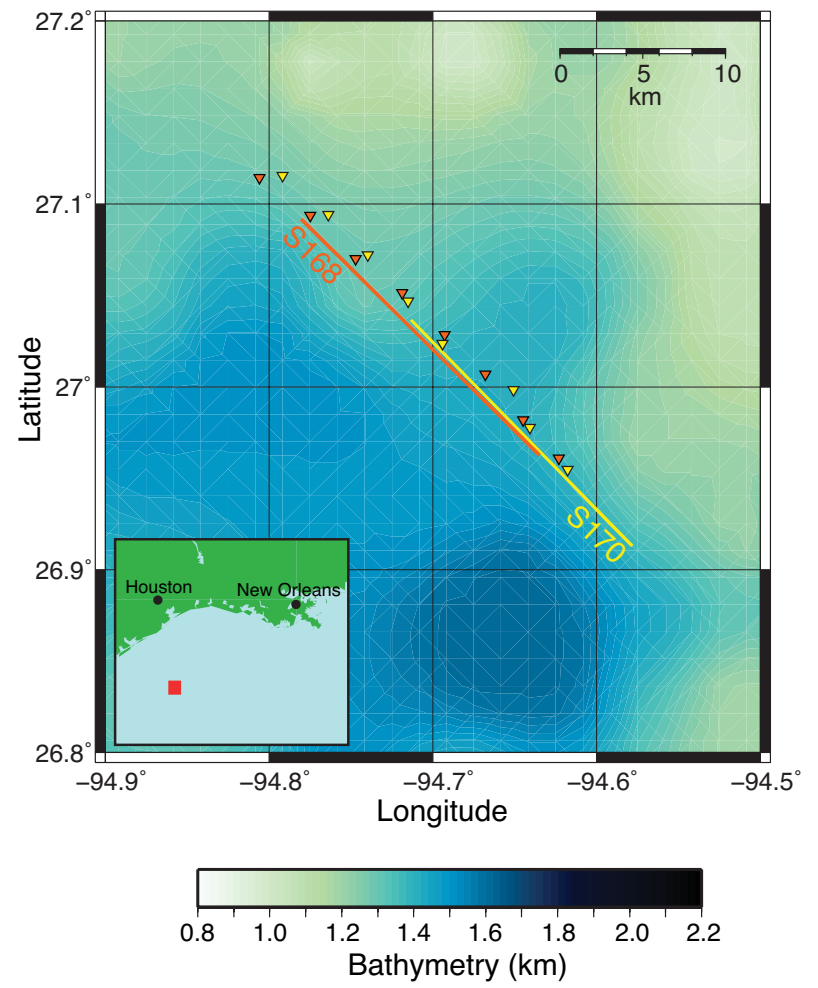

Fig. 1. Location map. Bathymetry has been interpolated from a $1^{\prime}$ grid (Smith and Sandwell, 1997) to $0.5^{\prime}$ grid spacing. Orange and yellow lines show locations of the CMP swaths; triangles show locations of the XBT casts.

even with this low fold we can discern strong continuous reflections in the water. We used a standard processing flow for the seismic data that included: applying a nominal geometry layout to the data, removal of coherent and random noise, filtering in the frequency-wavenumber domain, sorting into CMP gathers, band pass frequency filtering, normal moveout, median stacking, and extended Stolt (time) migration. Since there was little variation in the temperature profiles from the XBTs (see Fig. 2 for temperature profiles for swath S170; profiles for S168 looked very similar), we chose the sound speed profile from XBT 2886, swath S168 to create the smoothed root mean square (RMS) sound speed profile which was used to correct traces to zero offset (i.e., normal moveout) for all cables and sources. Although using RMS sound speeds as stacking velocities can result in problems in the earth where seismic speeds can vary greatly over short depth intervals, in the ocean sound speed variation is comparatively small, thus resulting in little difference between stacked images using XBT-derived RMS sound speeds and hand-picked stacking velocities (Fortin and Holbrook, 2009). In addition, we limited CMP gathers to near offsets which renders the stacked section relatively insensitive to small changes in stacking velocities. In the figures, two way time has been converted to depth assuming a constant 


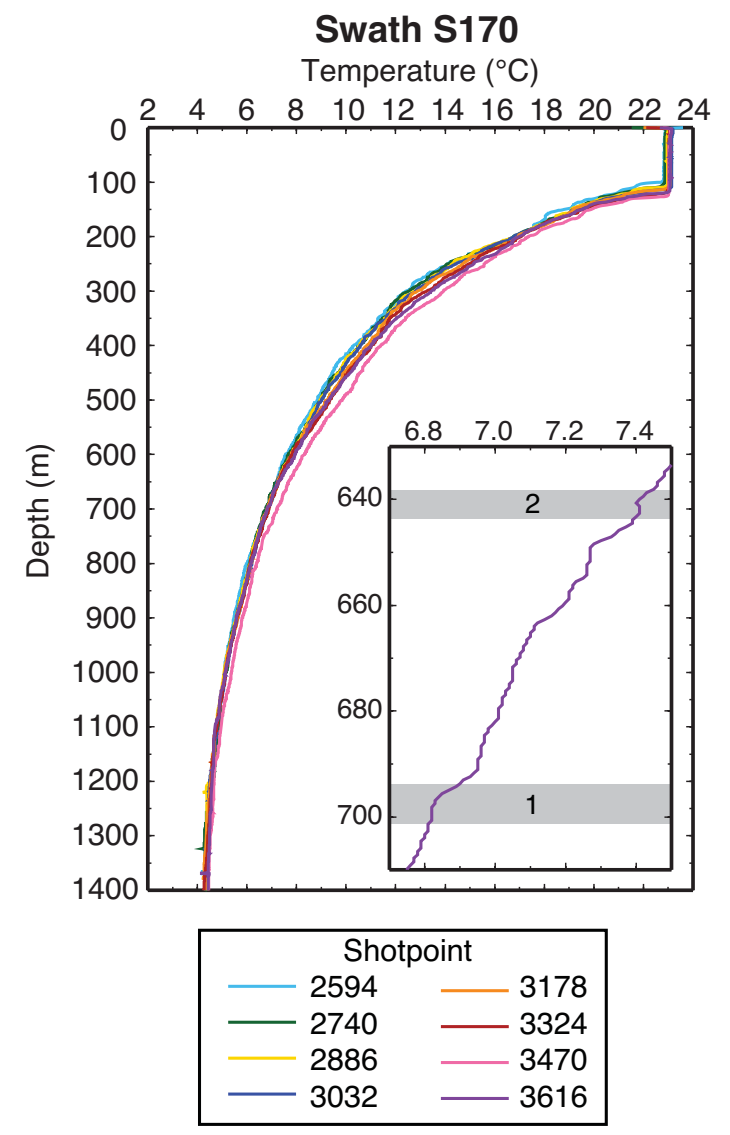

Fig. 2. Temperature profiles from XBT casts for swath S170. Inset shows blow-up of a portion of the profile for the XBT deployed nearest to the features shown in Fig. 6 (shotpoint 3616, $\sim 0.7 \mathrm{~km}$ from features in Fig. 6). Grey bars highlight a temperature stair step (feature 1) and a small inversion (feature 2) which correspond with the reflection surfaces shown in Fig. 6.

sound speed of $1500 \mathrm{~m} / \mathrm{s}$ corresponding to the average sound speed in the upper half of the water column.

We can estimate the resolution of our 2-D migrated images in the along-swath direction by considering the horizontal sample spacing. Sampling theory states that the smallest wavelength we can expect to distinguish, $\lambda_{n}$, (without taking noise into account) is two times the trace spacing (Sheriff and Geldart, 1995), which for our survey geometry results in $\lambda_{n}=12.5 \mathrm{~m}$. In the across-swath direction we must consider the Fresnel zone to limit our resolution since migration was only carried out in the along-swath direction. For water depths between 200 and $1200 \mathrm{~m}$, airgun sources with a dominant frequency of $50 \mathrm{~Hz}$, and assuming a sound speed of $1500 \mathrm{~m} / \mathrm{s}$ in the water, the first Fresnel zone, $R_{1}$, ranges from 55 to $134 \mathrm{~m}$. However, because the outer portion of the Fresnel zone contributes relatively little energy to the reflection signal at a detector, we can consider the effective Fresnel zone, $R_{1} / \sqrt{ } 2$ (Sheriff and Geldart, 1995), to limit the horizontal resolution in the across-swath direc- tion to 39-95 $\mathrm{m}$ over the same depth range. Thus, we can discern features with wavelengths less than half the swath width, depending on the depth of the reflector. 3-D processing can improve the signal to noise ratio of the entire image volume, and with trace interpolation in the cross-swath direction and trace padding to reduce edge effects, 3-D migration can improve the resolution limit in both along-swath and cross-swath directions to be on the order of twice the receiver spacing. Vertical resolution can be estimated as a quarter of the wavelength of the dominant frequency of the data (Widess, 1973). For our data, reflections have a frequency range of $30-100 \mathrm{~Hz}$ with the dominant frequency at approximately $50 \mathrm{~Hz}$. Assuming a sound speed of $1500 \mathrm{~m} / \mathrm{s}$ results in a vertical resolution of $\sim 8 \mathrm{~m}$.

Using XBT temperature profiles and the robust parabola model of Thacker (2007) for estimating salinity at depth in the Gulf of Mexico, we can produce density profiles at each XBT cast location. We assumed hydrostatic conditions for converting depth to pressure, accounting for the change in density at each depth increment, and, as the temperature profiles are relatively simple, we used a linear equation of state (Knauss, 1997) to calculate the density at depth. Isopycnals are plotted on top of that portion of the seismic image that overlaps with the XBT coverage in Fig. 3. The isopycnals are nearly horizontal across most of the image with a small downward dip beneath the XBT at shotpoint 3470 . The density was also used, along with sound speed, to calculate impedance and reflection coefficient (e.g., Ruddick et al., 2009), which was then convolved with an estimated source wavelet to produce a synthetic section (Fig. 4).

We have also performed a rudimentary stack of the entire 3-D data volume for swath S170. This initial processing consisted only of 3-D grid application, reduction of coherent and random noise, flex binning, normal moveout (using the same sound speed profile as in our 2-D processing), band pass frequency filtering and median stacking. Because 3-D migration has not yet been performed, the Fresnel zone limits the horizontal resolution of the resulting image volume to $\sim 55-135 \mathrm{~m}$ in all directions (for depths in the range of $\sim 200-1200 \mathrm{~m}$ ); however, we can still gain some insight into the potential of 3-D seismic data sets for interpretation of oceanic processes from this first pass through a basic 3-D data processing flow.

\section{Results and discussion}

In this data set, strong continuous reflections are concentrated in the upper $\sim 800 \mathrm{~m}$ (Figs. 3 and 5) with a few weaker but still discernable reflections as deep as $\sim 1100 \mathrm{~m}$. The reflections are largely subhorizontal and in many areas appear to follow isopycnals, which are also roughly horizontal with a small downward dip of $\sim 10-20$ m beneath XBT 3470 (Fig. 3). Examples of sloping reflections that may cross isopycnals can be seen between $\sim 1.5-3 \mathrm{~km}$ along the swath 


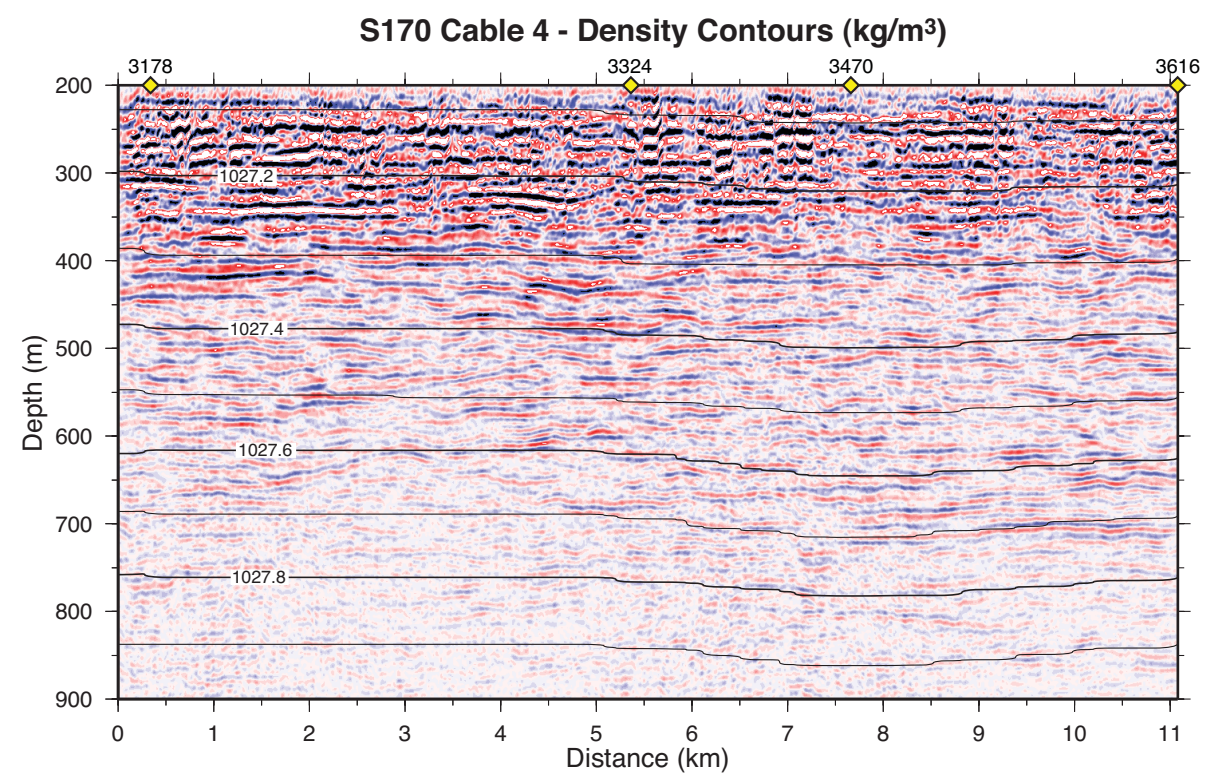

Fig. 3. The portion of the migrated image of swath S170, cable 4, source 1, that overlaps with XBT coverage is shown with isopycnals $\left(\mathrm{kg} / \mathrm{m}^{3}\right)$ plotted on top of the image. Yellow diamonds at the top indicate locations of XBT casts; numbers correspond to nearest shot point.

at depths of $\sim 600-650 \mathrm{~m}$, between $\sim 3-4.5 \mathrm{~km}$ along swath at depths of $\sim 500-550 \mathrm{~m}$, and between $\sim 9.2-10 \mathrm{~km}$ along the swath at a depth of $\sim 550 \mathrm{~m}$. A comparison between the temperature gradient $(\nabla T)$ and the migrated image (Fig. 4) shows that the brighter reflections in the seismic image can be associated with peaks in $\nabla T$, although the alignment of reflections with peaks in $\nabla T$ is not always perfect. The migrated image can also be compared to a synthetic section derived from the XBT data, shown on the right side of Fig. 4. The synthetic section shows the same first order trend of decreasing reflection amplitudes with increasing depth as seen in the data. In addition, many of the bright reflections in the synthetic section are aligned with bright reflections in the seismic data, although this is not always the case. This could be due to the fact that the boat from which the XBTs were cast was following the tail of the seismic streamer and so there was a time gap on the order of five minutes between when the shot was fired and when the XBT was cast at the approximate location of the shot. In that space of time the water continued to move, likely resulting in shifts in the location of reflectors. As can be seen in Fig. 1 there is also a spatial separation between the XBT casts and the seismic swath.

To investigate the source of the reflections we observe in our data, we must rely only on the XBT temperature profiles and the slope of the reflections relative to our estimated isopycnals as we lack coincident salinity profiles. Although the reflections generally appear to follow isopycnals (Fig. 3), the temperature profiles (Fig. 2) show only a few small inversions; they mostly consist of small stair steps with steep temperature gradients between thin layers of near-uniform

\section{Temperature Gradient}

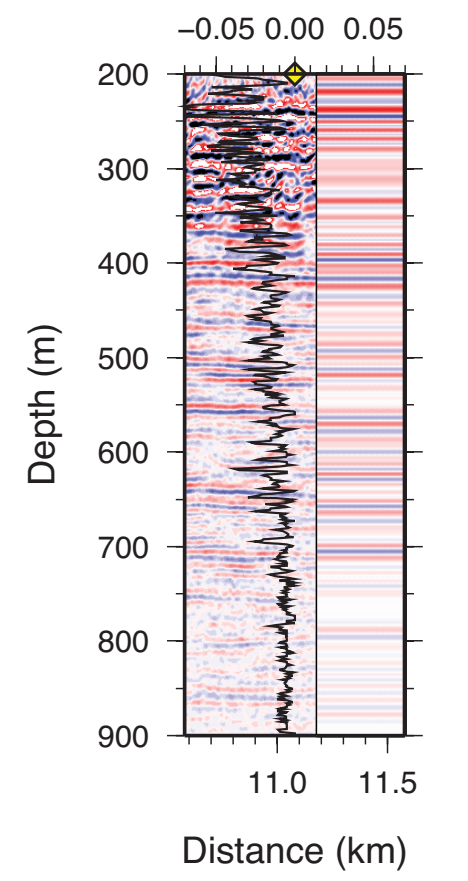

Fig. 4. Temperature gradient plotted on top of a portion of the migrated image of swath S170 (see Fig. 3) and a synthetic section. The synthetic section was calculated by convolving an average source wavelet with the reflection coefficient profile. Yellow diamond at the top indicates location of the XBT cast near shotpoint 3616, from which the reflection coefficient and temperature gradient have been derived. 


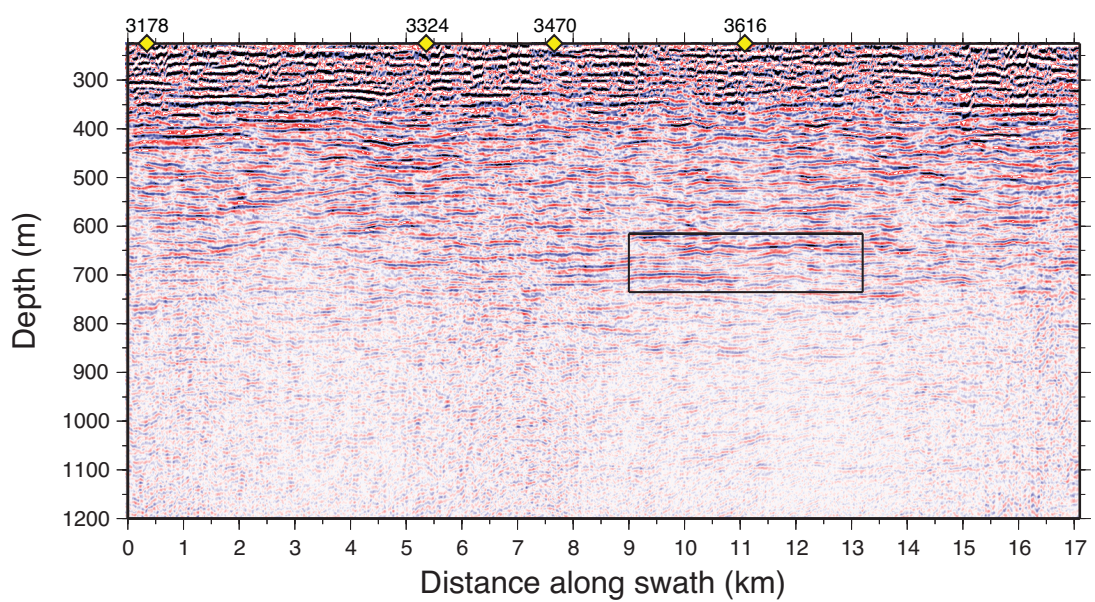

(a)

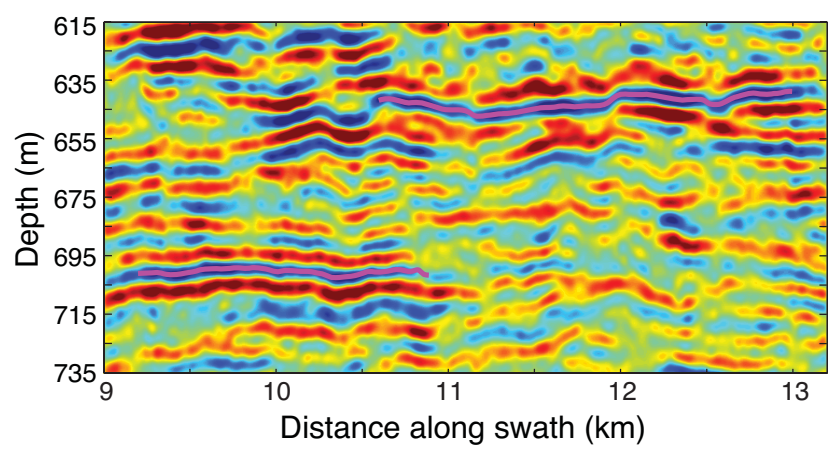

(b)

Fig. 5. (a) Migrated image of swath $\mathrm{S} 170$, cable 4, source 1. The images have been strongly smoothed but no relative gain control has been applied. Black box outlines region shown in (b). Yellow diamonds at top indicate locations of XBT casts; numbers correspond to nearest shot point. (b) Selected portion of the migrated image shown in (a). Two strong continuous reflections (highlighted by pink lines) were tracked across the swath to make the reflection surfaces shown in Fig. 6.

temperature. We therefore suggest that the reflections are not caused by fine structure from thermohaline intrusions but instead result from reversible fine structure caused by internal wave strain. Note that here we define "strain" following Thorpe (2005) as the vertical distance between two given isopycnal surfaces divided by their mean separation, and not in the formal sense of the strain tensor in an incompressible fluid. In this sense, a propagating internal wave causes zones of intensified or weakened "strain" (Thorpe, 2005, p. 60). The fine structure causing reflections in our data could also be a result of previous mixing with the boundaries between layers of well-mixed water perturbed by small-scale internal waves to produce the undulations we see in the reflections. This is not to say that any given reflection or reflection segment in our images could or should be associated with a single internal wave with a specific source. The wavefield at any one time is a superposition of many waves with peaks and troughs corresponding to locations of constructive interference of those waves. Therefore, some additional information on currents or other sources of internal waves is needed when interpreting the source of any particular reflection. For our data set, there is no additional oceanographic data available that could help us interpret a source for a reflection. However, for illustrative purposes, we examined and calculated the orientation of one wave peak in order to demonstrate the feasibility of obtaining such information from 3-D seismic reflection data.

In our current scheme using the 2-D lines it is necessary to find reflections that can be tracked across the entire swath (i.e., that are visible and continuous in all $16 \mathrm{mi}-$ grated images) over the same in-line distance interval in order to make a reflection surface. We chose two strong and relatively long reflections to make example surface plots. The black box in Fig. 5a outlines the area containing the chosen reflections, and Fig. 5b shows the tracked reflections within this box with the two chosen reflections highlighted in pink. At the depth of these reflections, $\sim 650 \mathrm{~m}$, the effective Fresnel zone width, representing the acrossswath resolution, is $\sim 70 \mathrm{~m}$. We refer to the deeper reflection as reflection 1 and the shallower reflection as reflection 2 . 


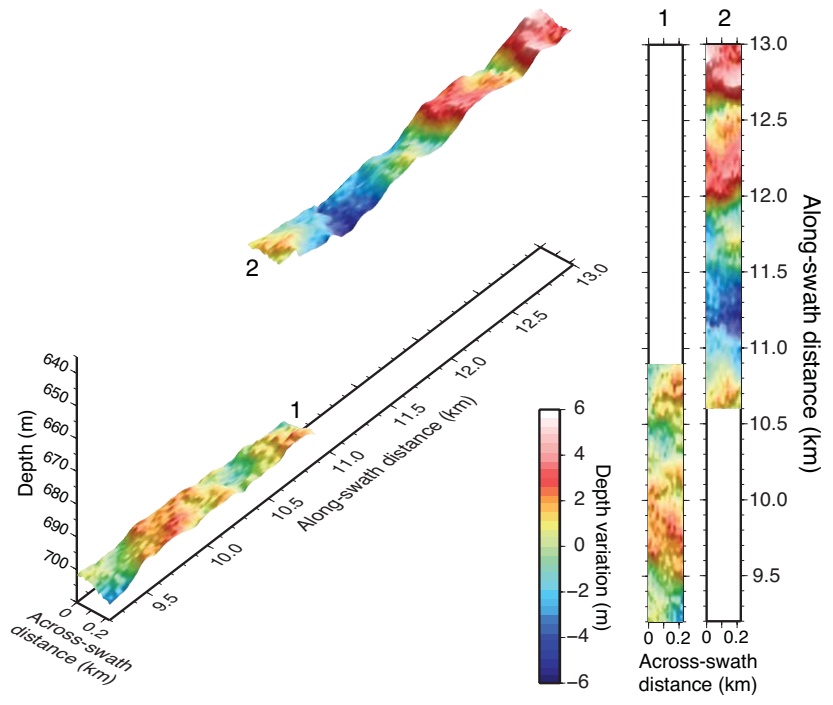

Fig. 6. Reflections 1 and 2, tracked in Fig. 5, are shown in 3-D perspective view (left) and 2-D map view (right).

Reflection 1 was tracked across the swath from along-swath distance $X=9.2$ to $10.9 \mathrm{~km}$; reflection 2 was tracked from $X=10.6$ to $13.0 \mathrm{~km}$. The "flying carpet" surface images that result from combining the $162-\mathrm{D}$ reflection tracks are shown in Fig. 6. Reflection 2 shows a greater degree of depth variability compared to reflection 1 , but the $3-\mathrm{D}$ nature of both reflections can be easily discerned. In general, the reflections display an egg carton-like surface of peaks and troughs, though some features have a more linear appearance.

Orientation of fine structure that is more linear in appearance can be determined by several means. As an example, we can fit a line by eye to the single wave crest in reflection 2 between 12 and $12.5 \mathrm{~km}$ in the along-swath direction. It should be noted that in this example we are not attempting to say anything about wave properties in the region but are investigating how we might fit a local feature. Knowing that the azimuth of the seismic swath is $\sim 134^{\circ}$ gives us an azimuth of $\sim 169 \pm 5^{\circ}$ for this wave crest. More rigorously, we can draw contours of wave relief and determine the orientation of each contour line segment, as shown in Fig. 7. Here we again focus on the portion of reflection 2 between 12 and $12.5 \mathrm{~km}$ along the swath. Before contouring, the data is first smoothed in the across-swath direction by applying a three point moving average filter. Orientations for the contour line segments are then weighted by line segment length and plotted in a rose diagram in Fig. 7b, which shows that the dominant direction of the feature is $\sim 30^{\circ}$ from the along-swath direction, or $\sim 164^{\circ}$ azimuth.

Another method for estimating reflection peak/trough orientation that does not depend on the chosen contouring algorithm is to fit a sinusoidal surface of the form
$G=A \sin (K x+L y+\phi)$

to the reflection of interest, where $\mathrm{A}$ is the amplitude, $\phi$ is the phase shift required to align the peaks (expressed as a fraction of the phase length), $K=|1 / \lambda| \cos \theta, L=|1 / \lambda| \sin \theta, \lambda$ is the wavelength, and $\theta$ is the orientation angle of the peaks. We began by fitting a surface by eye to the same portion of reflection 2 from 12 to $12.5 \mathrm{~km}$ along the swath, then adjusted $A, \phi, \lambda$ and $\theta$ until a reasonably good fit was obtained (Fig. 8, center). We then set $A$ to $1.55 \mathrm{~km}$, corresponding to the bestfitting value chosen by eye, and calculated model surfaces for a range of $\lambda$ and $\theta$, allowing the best-fitting phase shift $\phi$ to be chosen for each $(\lambda, \theta)$ pair. The model with the minimum error, calculated as the sum of the squared differences between the model surface and the reflection surface, is then our "best" model (Fig. 8, right). The error surface, plotted in Fig. 9 as a function of $\lambda$ and $\theta$, is relatively simple with a clear global minimum, indicating that this is a robust way to estimate the orientation of linear peaks or troughs in a reflection. Reflection peak/trough orientations for both the by eye fit and our best fit (azimuths of $171^{\circ}$ and $181^{\circ}$, respectively) compare reasonably well to the values obtained by adding up contour segment orientations or simply fitting a line by hand.

We note that lack of migration in the across-swath direction does not affect the determination of wave orientation by contour segments or sinusoidal surface fitting. Although the across-swath resolution of the image would be improved to $\sim 60 \mathrm{~m}$ with migration, this would have only a small effect on the location of the peaks and troughs and thus only a small effect on the orientation of the contour lines drawn or the relative error of different surface models. Therefore, measuring wave orientations is an important application of 3-D seismic oceanography images even when migration of the data is not performed. However, due to the increase in noise in the rose diagram and in the error surface for surface fitting when multiple features are included in the contouring, this method of determining wave orientation is best suited to easily discernable targets such as solitary waves and fronts.

The location, orientation, and timing of this survey was essentially random from an oceanographic perspective; both location and orientation were chosen to best suit the objectives of sub-seafloor exploration required by the oil company rather than to investigate any specific oceanic features, and timing was simply the result of the overall survey schedule rather than being set to coincide with tides or any seasonal events. However, in spite of this we are able to image some interesting oceanic features, namely, internal wave strains that cause temperature steps within the thermocline. The specific temperature variations that are the likely causes of reflections 1 and 2 are highlighted by the inset in Fig. 2, which shows a close up of a portion of the temperature profile from the XBT cast closest to the location of our chosen reflections, XBT 3616. Reflection 1 appears to be the result of a relatively large temperature step while reflection 2 was likely generated by a small temperature inversion. 


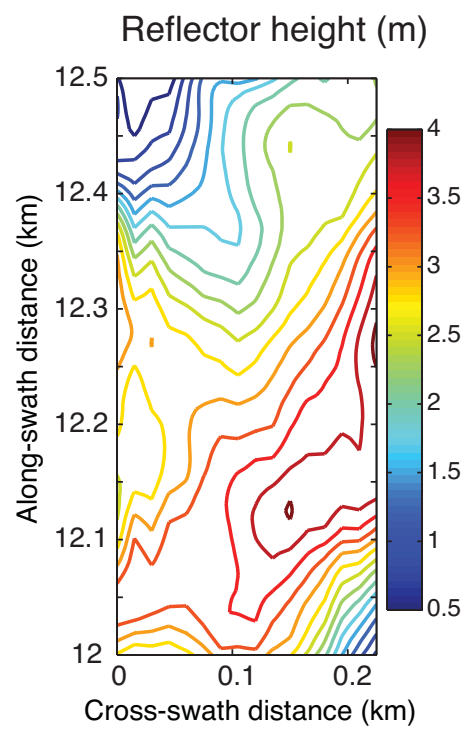

(a)

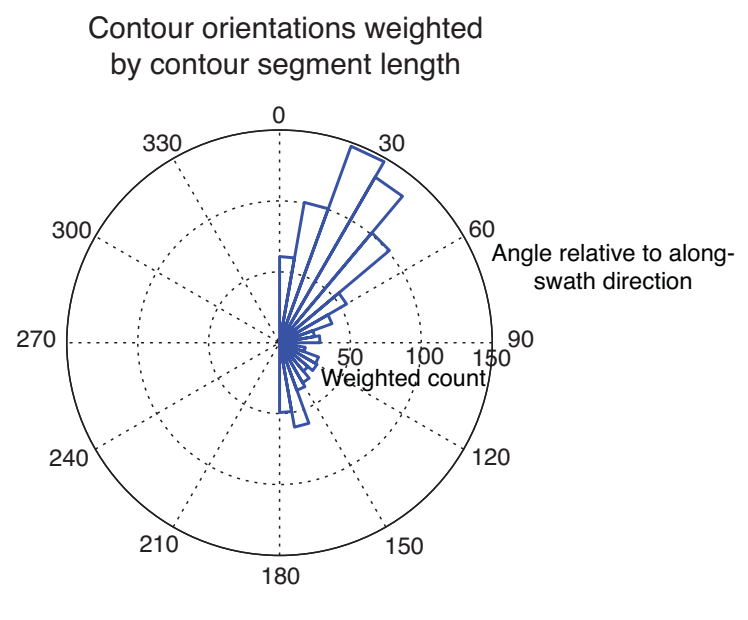

(b)

Fig. 7. Reflection peak/trough orientation relative to the swath can be obtained from a topographic contour plot of tracked reflections such as those shown in Fig. 6. (a) Contour plot of reflection relief for a portion of reflection 2 (see Fig. 6). (b) Rose diagram showing orientations of contour line segments relative to the along-swath direction. Histogram count has been weighted by contour line segment length.

Future work will include full 3-D processing of the data from both swaths including migration. This will place all CMP traces into grid boxes based on location, which will mitigate the streamer feathering problem and allow for greater and more uniform stacking fold. In general, once the stacking procedure has been optimized and 3-D migration has been performed, we expect to see an increase in the signal to noise ratio as well as an increase in our options for imaging and interpreting the structure of internal waves in this region. As an example of the type of diagram that can be made from the full 3-D stack volume to aid in interpretation, Fig. 10 shows a plot of vertical and horizontal (two way time) slices through part of the initial 3-D stack volume. In a given horizontal slice, reflection crossings appear as black bar-like features with wider bars that extend farther in the direction of the cross-line ordinals (equivalent to the alongswath direction) corresponding to flatter reflections (see inset in Fig. 10). In addition to making slices through the data volume, it should also be possible pick and plot horizons to make surfaces similar to those in Fig. 3, which were obtained from parallel 2-D images.

The complex 3-D internal wavefield we image here is not the ideal target for the feature orientation calculation we have presented; attempting to interpret a source for any particular bump in a reflection without additional oceanographic information is difficult at best. In addition, due to the time required for the seismic vessel to turn and perform side-by-side swaths and the dynamic nature of ocean fine structure, the width of the 3-D image volume is limited to a single swath ( $225 \mathrm{~m}$ in this case). For these reasons we suggest that the

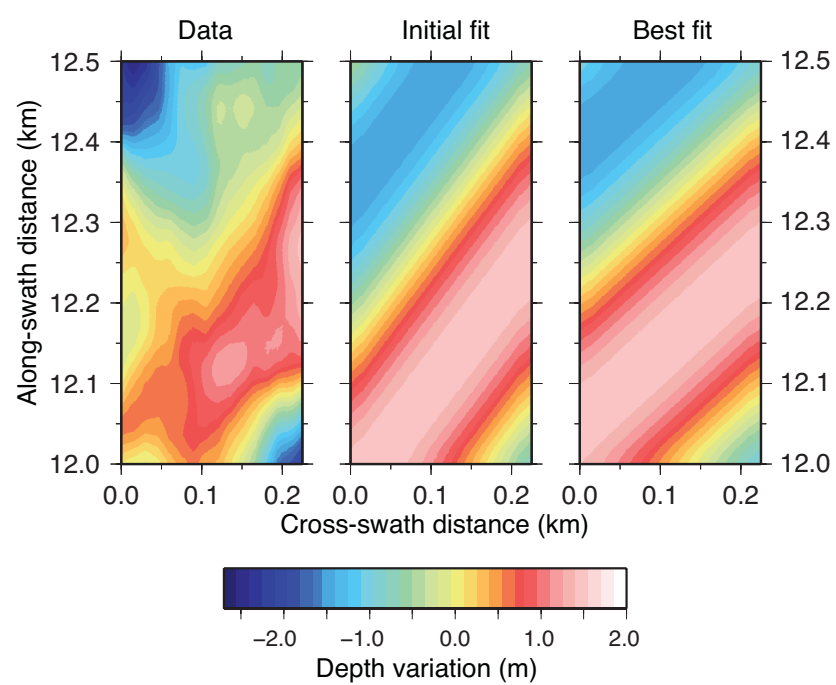

Fig. 8. A second way of determining reflection peak/trough orientation is to fit a sinusoidal surface to the reflection data. The data (same portion of reflection 2 as shown in Fig. 7) is plotted on the left. In the center is a sinusoidal surface fit obtained by adjusting surface parameters by hand until a fit is obtained that looks good to the eye. This gives an orientation angle of $37^{\circ}$ (corresponding to an azimuth of $171^{\circ}$ ), wavelength of $0.45 \mathrm{~km}$ and phase shift of 0.25 times the phase length, where the orientation angle is defined as in Fig. 7. On the right is the "best" fit (fit with minimum error). This surface has an orientation angle of $47^{\circ}$ (corresponding to an azimuth of $181^{\circ}$ ), wavelength of $0.505 \mathrm{~km}$, and phase shift of 0.7 times the phase length. 

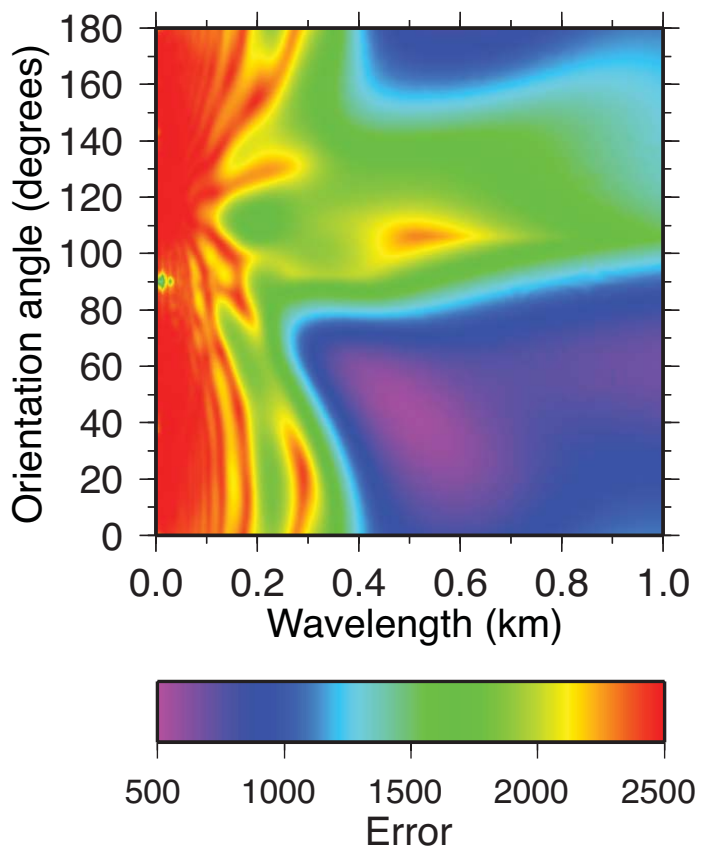

Fig. 9. Error (sum of squared differences) surface as a function of wavelength and orientation angle (defined as in Fig. 7) for sinusoidal surface fitting of the reflection portion shown in Fig. 8.

best targets for this method are well-defined features such as solitary waves, fronts, eddies, lee waves, or internal waves from a known or postulated specific source. When focusing on these kinds of features, time-lapse imaging could also be valuable. The seismic ship could pass over the same area hours or days later and changes in the reflection fabric over that time could then be examined. Our data set from the Gulf of Mexico, though not ideal, illustrates the potential of 3-D seismic imaging of ocean fine structure when applied to a suitable target.

\section{Conclusions}

We have performed 2-D and basic, preliminary 3-D processing of an industry 3-D multichannel seismic data set provided by an oil company for oceanographic analysis. The data set includes 8 XBT casts for each of two 3-D seismic swaths, allowing us to use a local sound speed profile in our seismic data processing. The 2-D processing of one swath resulted in 16 parallel seismic images. Subhorizontal reflections are common in the images down to about $900 \mathrm{~m}$ depth. The larger peaks in the XBT temperature gradient and synthetic seismic section derived from the temperature profile and an empirical salinity profile correspond well with the locations of stronger reflections in the seismic image. In some places, reflections can be seen to cross the roughly horizontal isopycnals while in other areas they appear to follow the isopycnals. Temperature profiles show no evidence for ther-

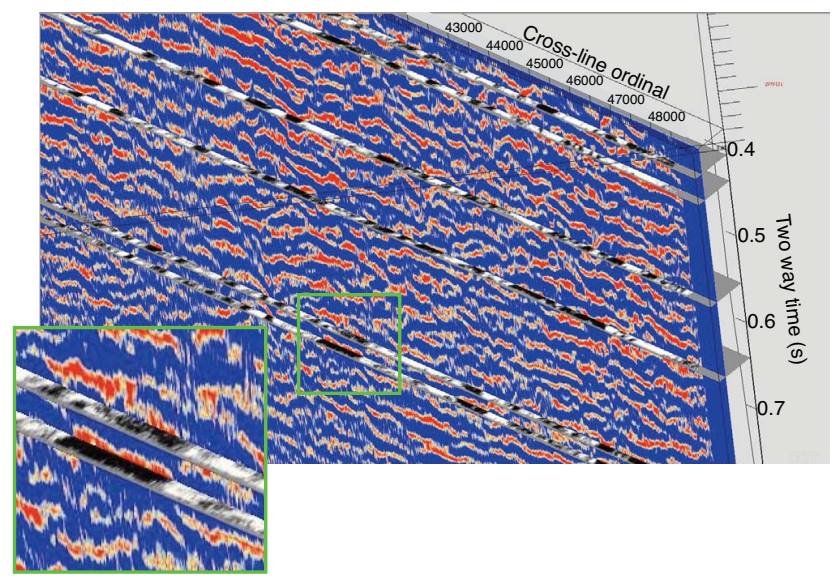

Fig. 10. 3-D view of vertical (color) and horizontal (black and white) slices through the 3-D stack volume. Data is un-migrated and a uniform gain has been applied. Cross-line ordinals denote the grid boxes in the along-swath direction. Green box outlines zoomed-in region shown in the inset.

mohaline intrusions, so we therefore interpret the reflections we see in our data to be caused by reversible fine structure from internal wave strains (with "strain" defined as the vertical distance between two given isopycnal surfaces divided by their mean separation).

To illustrate the utility of 3-D seismic data in studying the ocean, we selected two relatively strong and laterally continuous reflections that could be tracked in each of the $162-\mathrm{D}$ images. Combining the tracks produced 3-D reflection surface images that reveal the 3-D nature of the fine structure captured by the seismic data. Reflection surfaces display a complex egg carton-like distribution of peaks and troughs. The orientation of the more linear wave crests and troughs can be determined relative to the known azimuth of the seismic swath by plotting a histogram of wave height contour line orientations or, more robustly, by fitting a sinusoidal surface to the reflection. Results of a first pass through a basic 3-D processing flow provide additional insight into the potential of 3-D data to aid in the interpretation of seismic images of ocean features such as internal wave strains. We expect full, optimized 3-D processing of the data to result in improved signal to noise ratio in the images and increased options for imaging and interpreting 3-D structures in the ocean.

This work demonstrates the viability of 3-D imaging of ocean structures as well as the feasibility of obtaining quantitative measures such as the orientation of structures from these images. The setting of the data set used in this study was essentially random as it was chosen based on industry interest in a petroleum prospect rather than on oceanographic targets. However, despite this the images of fine structure we obtained suggest that more dramatic results can be obtained by applying 3-D seismic imaging to specific oceanic targets 
such as solitary waves, eddies, fronts, and lee waves. Indeed, such focused targets that can be characterized by $\sim 225-\mathrm{m}-$ wide image slices are the best targets for this method as multiple swaths cannot be combined into a single image volume due to the time gap between swaths (on the order of hours) and the dynamic nature of oceanic features. However, multiple swaths over the same area could be compared to obtain time-lapse images of the target for studying the evolution of a feature over hours or days.

Acknowledgements. We would like to thank all the commenters for their thoughtful and helpful insights. 2-D and 3-D seismic processing was carried out using the Omega2 program package from Western Geco. Figures shown in this paper were created using the Generic Mapping Tools software (Wessel and Smith, 1991), Matlab, and The Kingdom Suite.

Edited by: M. Hecht

\section{References}

Biescas, B., Sallarès, V., Pelegrí, J. L., Machín, F., Carbonell, R., Buffett, G., Dañobeitia, J. J., and Calahorrano, A.: Imaging meddy finestructure using multichannel seismic reflection data, Geophys. Res. Lett., 35, L11609, doi:10.1029/2008GL033971, 2008.

Cooper, C., Forristall, G. Z., and Joyce, T. M.: Velocity and hydrographic structure of two Gulf of Mexico warm-core rings, J. Geophys. Res., 95(C2), 1663-1679, 1990.

Egbert, G. D., Bennett, A. F., and Foreman, M. G. G.: TOPEX/POSEIDON tides estimated using a global inverse model, J. Geophys. Res., 99(C12), 24821-24852, 1994.

Farmer, D. and Armi, L.: The generation and trapping of solitary waves over topography, Science, 283(5399), 188-190, 1999.

Fortin, W. F. J. and Holbrook, W. S.: Sound speed requirements for optimal imaging of seismic oceanography data, Geophys. Res. Lett., 36, L00D01, doi:10.1029/2009GL038991, 2009.

Holbrook, W. S., Paramo, P., Pearse, S., and Schmitt, R. W.: Thermohaline fine structure in an oceanographic front from seismic reflection profiling, Science, 301, 821-824, 2003.

Ikeda, M. and Emery, W. J.: Satellite observations and modeling of meanders in the California Current system off Oregon and northern California, J. Phys. Oceanogr., 14(9), 1434-1450, 1984.

Klymak, J. M. and Moum, J. N.: Oceanic Isopycnal Slope Spectra: Part I - Internal Waves, J. Phys. Oceanogr., 37(5), 1215-1231, 2007.
Knauss, J. A.: Introduction to Physical Oceanography, Second Edition, Waveland Press, Inc., Long Grove, Illinois, USA, 1997.

Nakamura, Y., Noguchi, T., Tsuji, T., Itoh, S., Niino, H., and Matsuoka, T.: Simultaneous seismic reflection and physical oceanographic observations of oceanic fine structure in the Kuroshio extension front, Geophys. Res. Lett., 33, L23605, doi:10.01029/2006GL027437, 2006.

Nandi, P., Holbrook, W. S., Pearse, S., Paramo, P., and Schmitt, R. W.: Seismic reflection imaging of water mass boundaries in the Norwegian Sea, Geophys. Res. Lett., 31, L23311, doi:10.1029/2204GL021325, 2004.

Ruddick, B., Song, H., Dong, C., and Pinheiro, L.: Water Column Seismic Images as Maps of Temperature Gradient, Oceanography, 22(1), 192-205, 2009.

Rudnick, D. L. and Ferrari, R.: Compensation of horizontal temperature and salinity gradients in the ocean mixed layer, Science, 283, 526-529, 1999.

Rudnick, D. L., Boyd, T. J., Brainard, R. E., Carter, G. S., Egbert, G. D., Gregg, M. C., Holloway, P. E., Klymak, J. M., Kunze, E., Lee, C. M., Levine, M. D., Luther, D. S., Martin, J. P., Merrifield, M. A., Moum, J. N., Nash, J. D., Pinkel, R., Rainville, L., and Sanford, T. B.: From tides to mixing along the Hawaiian Ridge, Science, 301, 355-357, 2003.

Sheriff, R. E. and Geldart, L. P.: Exploration Seismology, Cambridge University Press, Cambridge, United Kingdom, 1994.

Smith, W. H. F. and Sandwell, D. T.: Global seafloor topography from satellite altimetry and ship depth soundings, Science, 277, 1957-1962, 1997.

Thacker, W. C.: Estimating salinity to complement observed temperature: 1. Gulf of Mexico, J. Marine Syst., 65, 224-248, 2007.

Thorpe, S. A.: The Turbulent Ocean, Cambridge University Press, Cambridge, United Kingdom, 2005.

Tsuji, T., Noguchi, T., Niino, H., Matsuoka, T., Nakamura, Y., Tokuyama, H., Kuramoto, S., and Bangs, N.: Twodimensional mapping of fine structures in the Kuroshio Current using seismic reflection data, Geophys. Res. Lett., 32, L14609, doi:10.1029/2005GL023095, 2005.

Wessel, P. and Smith, W.: Free software helps maps and display data, EOS, 72(41), 441-446, 1991.

Widess, M.: How thin is a thin bed?, Geophysics, 38, 1176-1180, 1973.

Wiebe, P. H., Stanton, T. K., Benfield, M. C., Mountain, D. G., and Greene, C. H.: High-frequency acoustic volume backscattering in the Georges Bank coastal region and its interpretation using scattering models, J. Ocean. Eng., 22(3), 445-464, 1997. 